Internat. J. Math. \& Math. Sci.

Vol. 23, No. 5 (2000) 361-365

S0161171200001988

(C) Hindawi Publishing Corp.

\title{
ALMOST AUTOMORPHIC SOLUTIONS OF SOME DIFFERENTIAL EQUATIONS IN BANACH SPACES
}

\author{
GASTON MANDATA N'GUEREKATA
}

(Received 12 November 1997 and in revised form 2 March 1998)

\begin{abstract}
We discuss the conditions under which bounded solutions of the evolution equation $x^{\prime}(t)=A x(t)+f(t)$ in a Banach space are almost automorphic whenever $f(t)$ is almost automorphic and $A$ generates a $C_{0}$-group of strongly continuous operators. We also give a result for asymptotically almost automorphic solutions for the more general case of $x^{\prime}(t)=A x(t)+f(t, x(t))$.
\end{abstract}

Keywords and phrases. Almost automorphic functions, mild solutions, generator of a $C_{0}{ }^{-}$ group, linear operators.

2000 Mathematics Subject Classification. Primary 34G10.

1. Introduction. Let $A$ generate a $C_{0}$-group of strongly continuous operators $T(t)$, $t \in \mathbb{R}$ on a Banach space $X$. Let $f \in L^{\infty}(\mathbb{R} ; X)$. A basic unsolved problem is: what is the structure of bounded (on $\mathbb{R}$ ) mild solutions of $x^{\prime}(t)=A x(t)+f(t)$ ? Classically results go back to Ordinary Differential Equations (when dimension of $X$ is finite), and one sought solutions $x(t)$ such that $x(t)-y(t) \rightarrow 0$ as $t \rightarrow \infty$, when either $y(t)$ is a constant or a periodic function of time. In the evolution context of $x^{\prime}=A x+f$, much has been written on asymptotically constant or periodic solutions. Several authors extended these ideas to almost periodic solutions (when $f$ is almost periodic). Our main result (Theorem 1.6) is inspired by the interesting work of Goldstein [3]. We are actually concerned with the more general case of almost automorphic, and when bounded solutions are almost automorphic. We also give a new result (Theorem 1.7) concerning mild solutions of the equation $x^{\prime}(t)=A x(t)+f(t, x(t))$ which approach almost automorphic functions at infinity under specific conditions on the function $f(t, x)$. See also [6] for another comparable situation.

Let $X$ be a Banach space equipped with the topology norm and $\mathbb{R}=(-\infty, \infty)$ the set of real numbers. Let us first recall some definitions.

DEFINITION 1.1 (Bochner). A continuous function $f: \mathbb{R} \rightarrow X$ is said to be almost automorphic if and only if, from any sequence of real numbers $\left(s_{n}^{\prime}\right)_{n=1}^{\infty}$, we can subtract a subsequence $\left(s_{n}\right)_{n=1}^{\infty}$ such that: $\lim _{n \rightarrow \infty} f\left(t+s_{n}\right)=g(t)$ exists for each real number $t$, and $\lim _{n \rightarrow \infty} g\left(t-s_{n}\right)=f(t)$ for each $t$.

DEFINITION 1.2 [4]. A continuous function $f: \mathbb{R}^{+} \rightarrow X$ is said to be asymptotically almost automorphic if and only if there exists an almost automorphic function $g$ : $\mathbb{R} \rightarrow X$ and a continuous function $h: \mathbb{R}^{+} \rightarrow X$ with $\lim _{t \rightarrow \infty}\|h(t)\|=0$ and such that $f(t)=g(t)+h(t)$ for each $t \in \mathbb{R}^{+}$. 
DEFINITION 1.3. A Banach space $X$ is said to be perfect if and only if every bounded function $u: \mathbb{R} \rightarrow X$ with an almost automorphic derivative $u^{\prime}(t)$ is necessarily almost automorphic.

REMARK 1.4. Uniformly convex Banach spaces are nice examples of perfect Banach spaces (see [10, Theorem 1.4]).

We consider the evolution equation

$$
x^{\prime}(t)=A x(t)+f(t), \quad t \in \mathbb{R} .
$$

THEOREM 1.5. Let $X$ be a perfect Banach space. Let $A$ be a bounded linear operator $X \rightarrow X$ and $f: \mathbb{R} \rightarrow X$ an almost automorphic function. Then any bounded strong solution of (1.1) is almost automorphic if we assume that there exists a finite-dimensional subspace $X_{1}$ of $X$ such that

( $\alpha) A x(0) \in X_{1}$,

( $\beta)\left(e^{t A}-I\right) f(s) \in X_{1}$ for any $s, t \in \mathbb{R}$,

( $\gamma) e^{t A} u \in X_{1}$ for any $t \in \mathbb{R}$ and for any $u \in X_{1}$.

Proof. Let $P$ be the projection of $X$ onto $X_{1}$; such $P$ always exists (cf. [7]) and possesses the following properties:

(1) $X=X_{1} \oplus \operatorname{ker}(P)$, where $\operatorname{ker}(P)$ is the kernel of the operator $P$,

(2) $P$ is bounded on $X$.

If we put $Q=I-P$, then it is easy to verify that $Q^{2}=Q$ on $X$ and $Q u=0$ for any $u \in X_{1}$. Now if $x(t)$ is a bounded solution of (1.1), then we can write it as

$$
x(t)=x_{1}(t)+x_{2}(t)
$$

with $x_{1}(t)=P x(t) \in X_{1}$ and $x_{2}(t)=Q x(t) \in \operatorname{ker}(P)$.

Since $x(t)$ is bounded on $\mathbb{R}$, it is clear that both $x_{1}(t)$ and $x_{2}(t)$ are also bounded on $\mathbb{R}$. On the other hand, we have

$$
x^{\prime}(t)=x_{1}^{\prime}(t)+x_{2}^{\prime}(t)=A x_{1}(t)+A x_{2}(t)+P f(t)+Q f(t), \quad t \in \mathbb{R} .
$$

But $x(t)$ has the well-known Lagrange representation:

$$
\begin{aligned}
x(t) & =e^{t A} x(0)+\int_{0}^{t} e^{(t-s) A} f(s) d s \\
& =e^{t A} x(0)+\int_{0}^{t} f(s) d s+\int_{0}^{t}\left(e^{(t-s) A}-I\right) f(s) d s .
\end{aligned}
$$

By assumption $(\beta)$, we deduce that $\int_{0}^{t}\left(e^{(t-s) A}-I\right) f(s) d s$ is in $X_{1}$, so that if we apply $Q$ to both sides of (1.4), we get

$$
x_{2}(t)=Q e^{t A} x(0)+Q \int_{0}^{t} f(s) d s=Q e^{t A} x(0)+\int_{0}^{t} Q f(s) d s,
$$

consequently

$$
x_{2}^{\prime}(t)=Q e^{t A} A x(0)+Q f(t)=Q f(t)
$$

using conditions $(\alpha)$ and $(\gamma)$. 
It is clear that $Q f(t)$ and thus $x_{2}^{\prime}(t)$ is almost automorphic (see [9, page 586]). Since $x_{2}(t)$ is bounded, then it is almost automorphic for we are in a perfect Banach space.

Now if we apply $P$ to both sides of (1.3), we get in the finite-dimensional space $X_{1}$ the differential equation

$$
x_{1}^{\prime}(t)=P A x_{1}(t)+P A x_{2}(t)+P^{2} f(t)+P Q f(t), \quad t \in \mathbb{R} .
$$

Since the function $g(t) \equiv P^{2} f(t)+P Q f(t)$ is almost automorphic and $P A$ is a bounded linear operator, we deduce that $x_{1}(t)$ is almost automorphic [9, Theorem 3]. Finally, $x(t)$ is almost automorphic as the sum of two almost automorphic functions.

Theorem 1.5 can be generalized to the case of unbounded operator $A$ as follows.

THEOREM 1.6. In a perfect Banach space $X$, let $A$ generate a $C_{0}$-group of strongly continuous linear operators $T(t), t \in \mathbb{R}$. Assume that there exists a finite-dimensional subspace $X_{1}$ of $X$ such that:

( $\alpha) A x(0) \in X_{1}$,

$\left(\beta^{\prime}\right)(T(t)-I) f(s) \in X_{1}$ for any $s, t \in \mathbb{R}$,

( $\gamma$ ) $T(t) u \in X_{1}$ for any $t \in \mathbb{R}$ and any $u \in X_{1}$.

Then every bounded solution of (1.1) is almost automorphic.

Proof. We just follow the proof of Theorem 1.5 with the appropriate modifications. Here solutions are written as $x(t)=T(t) x(0)+\int_{0}^{t} T(t-s) f(s) d s$.

We return now to a general (not necessarily perfect) Banach space $X$. We state and prove the following theorem.

THEOREM 1.7. Let $A$ be a (possibly unbounded) linear operator which is the generator of a $C_{0}$-group of strongly continuous linear operators $T(t), t \in \mathbb{R}$ such that $T(t) x: \mathbb{R} \rightarrow X$ is almost automorphic for each $x \in X$. Consider the differential equation

$$
x^{\prime}(t)=A x(t)+f(t, x(t)),
$$

where $f(t, x): \mathbb{R} \times X \rightarrow X$ is strongly continuous with respect to jointlyt and $x$ and such that $\|f(t, x)-f(t, y)\| \leq L\|x-y\|$ for any $t \in \mathbb{R}, x, y \in X$, and $\int_{0}^{\infty}\|f(t, 0)\| d t<\infty$.

Then every mild solution $x(t)$ of (1.8) with $\int_{0}^{\infty}\|x(t)\| d t<\infty$ is asymptotically almost automorphic.

Proof. Let $x: \mathbb{R}^{+} \rightarrow X$ be a mild solution of (1.8). Then we have

$$
x(t)=T(t) x(0)+\int_{0}^{t} T(t-s) f(s, x(s)) d s .
$$

We claim that $\int_{0}^{\infty} T(-s) f(s, x(s)) d s$ exists in $X$ (in Bochner's sense). Indeed, since $T(t)$ is almost automorphic for each $x \in X$, then

$$
\sup _{t \in \mathbb{R}}\|T(t) x\|<\infty \quad \text { for each } x \in X .
$$

Consequently

$$
\sup _{t \in \mathbb{R}}\|T(t)\|=M<\infty,
$$


by the uniform boundedness principle. Let us write

$$
\int_{0}^{\infty} T(-s) f(s, x(s)) d s=\int_{0}^{\infty} T(-s)(f(s, x(s))-f(s, 0)) d s+\int_{0}^{\infty} T(-s) f(s, 0) d s,
$$

then we get the inequality

$$
\left\|\int_{0}^{\infty} T(-s) f(s, x(s)) d s\right\| \leq M\left(L \int_{0}^{\infty}\|x(s)\| d s+\int_{0}^{\infty}\|f(s, 0)\| d s\right)<\infty .
$$

Now the continuous function $F: \mathbb{R} \rightarrow X$ defined by

$$
F(t)=\int_{0}^{\infty} T(t-s) f(s, x(s)) d s=T(t) \int_{0}^{\infty} T(-s) f(s, x(s)) d s
$$

is almost automorphic; therefore $V(t)=T(t) x(0)+F(t)$ is also almost automorphic. Let us consider the continuous function $W: \mathbb{R}^{+} \rightarrow X$

$$
W(t)=-\int_{t}^{\infty} T(t-s) f(s, x(s)) d s .
$$

If we use the same computation as for $F(t)$ in (1.14), we get

$$
\|W(t)\| \leq M\left(L \int_{t}^{\infty}\|x(s) d s\|+\int_{t}^{\infty}\|f(s, 0) d s\|\right)
$$

which shows that $\lim _{t \rightarrow \infty}\|W(t)\|=0$.

Finally $x(t)=V(t)+W(t), t \in \mathbb{R}^{+}$is asymptotically almost automorphic.

REMARK 1.8. (1) An example of Theorem 1.5 (occurring in Sturm-Liouville theory, for instance) is when $X$ is a Hilbert space and $A \varphi_{n}=\lambda_{n} \varphi_{n}$ for $\left\{\varphi_{n}: n=1,2, \ldots\right\}$ an orthonormal basis and $\left|\operatorname{Re}\left(\lambda_{n}\right)\right| \leq M$ for all $n$. For $X_{1}$, one may take $X_{1}=$ $\operatorname{span}\left\{\varphi_{1}, \ldots, \varphi_{N}\right\}$ (for any $N$ ) and assume $f \in L^{\infty}\left(\mathbb{R}, X_{1}\right)$.

(2) An example of operator $A$ satisfying the hypothesis of Theorem 1.7 is the above example with $A^{*}=-A$, i.e., $M=0$.

ACKNOWLEDGEMENTS. The present research was supported by a research grant from Morgan State University. The author is very grateful to the referee for his/her useful suggestions.

\section{REFERENCES}

[1] S. Bochner, Uniform convergence of monotone sequences of functions, Proc. Nat. Acad. Sci. U.S.A. 47 (1961), 582-585. MR 23\#A3390. Zbl 103.05304.

[2] Continuous mappings of almost automorphic and almost periodic functions, Proc. Nat. Acad. Sci. U.S.A. 52 (1964), 907-910. MR 29\#6252. Zbl 134.30102.

[3] J. A. Goldstein, Convexity, boundedness, and almost periodicity for differential equations in Hilbert space, Internat. J. Math. Math. Sci. 2 (1979), no. 1, 1-13. MR 80e:34040. Zbl 397.34041.

[4] G. M. N'Guérékata, Sur les solutions presqu'automorphes d'équations différentielles abstraites, Ann. Sci. Math. Québec 5 (1981), no. 1, 69-79. MR 82h:34085. Zbl 494.34045. 
[5] , Quelques remarques sur les fonctions asymptotiquement presque automorphes, Ann. Sci. Math. Québec 7 (1983), no. 2, 185-191. MR 84k:43009. Zbl 524.34064.

[6] _ An asymptotic theorem for abstract differential equations, Bull. Austral. Math. Soc. 33 (1986), no. 1, 139-144. MR 87c:34126. Zbl 581.34029.

[7] M. Schechter, Principles of Functional Analysis, Academic Press [Harcourt Brace Jovanovich Publishers], New York, 1973. MR 57\#7085.

[8] W. A. Veech, Almost automorphic functions on groups, Amer. J. Math. 87 (1965), 719-751. MR 32\#4469. Zbl 137.05803.

[9] S. Zaidman, Almost automorphic solutions of some abstract evolution equations, Istit. Lombardo Accad. Sci. Lett. Rend. A 110 (1976), no. 2, 578-588 (1977). MR 58\#6593. Zbl 374.34042.

[10] M. Zaki, Almost automorphic solutions of certain abstract differential equations, Ann. Mat. Pura Appl. (4) 101 (1974), 91-114. MR 51\#1059. Zbl 304.42028.

N'Guerekata: Department of MAthematics, Morgan State University, Cold Spring LANE AND HILLEN ROAD, BALTIMORE, MD 21251, USA 


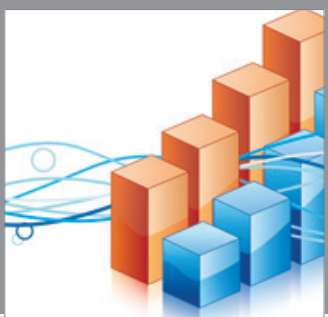

Advances in

Operations Research

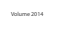

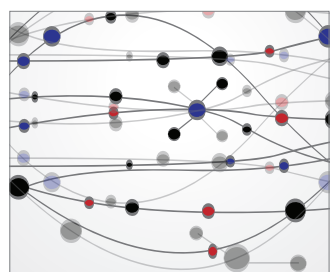

\section{The Scientific} World Journal
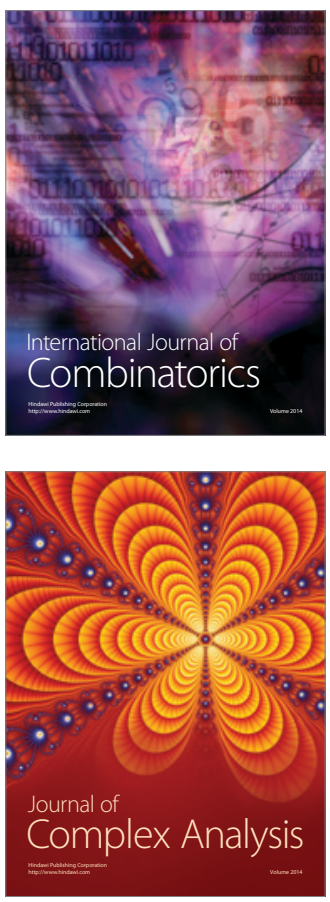

International Journal of

Mathematics and

Mathematical

Sciences
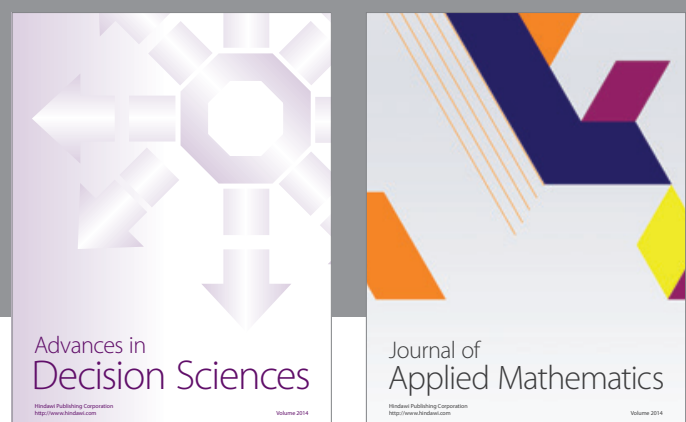

Journal of

Applied Mathematics
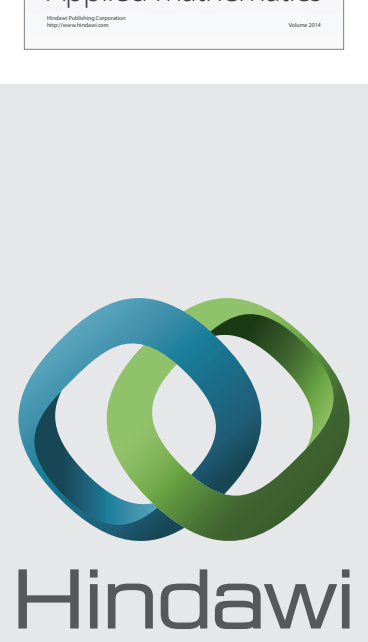

Submit your manuscripts at http://www.hindawi.com
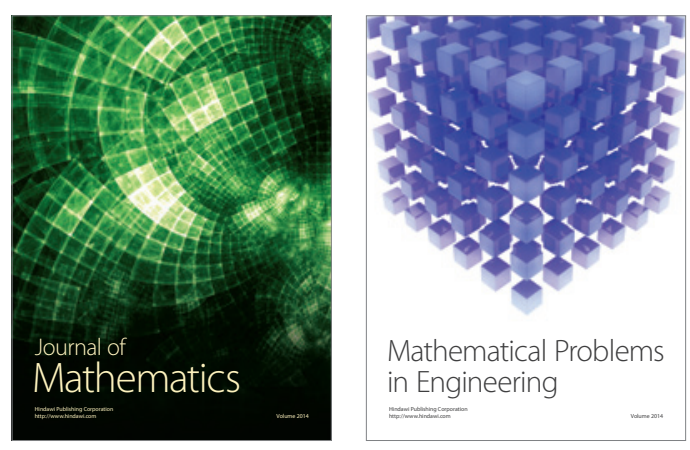

Mathematical Problems in Engineering
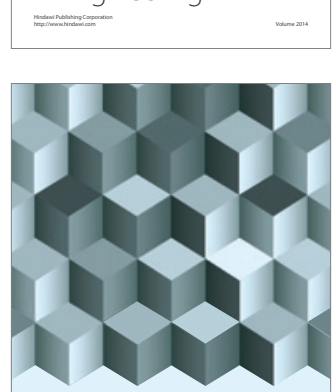

Journal of

Function Spaces
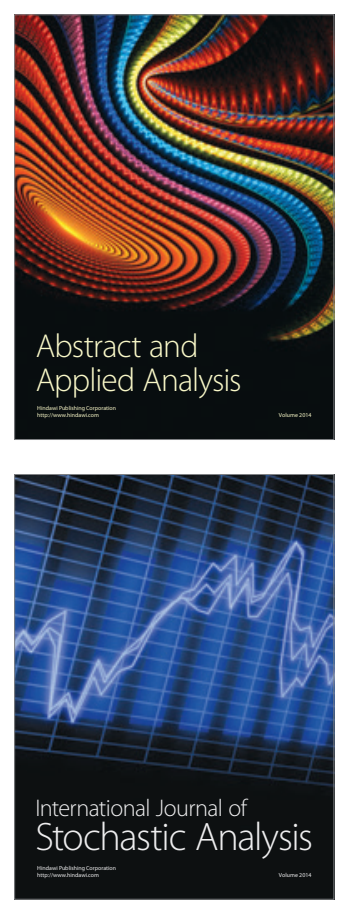

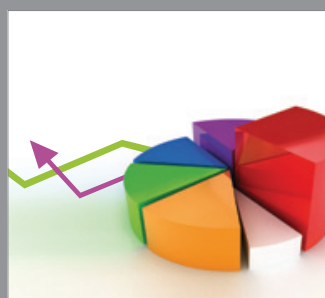

ournal of

Probability and Statistics

Promensencen
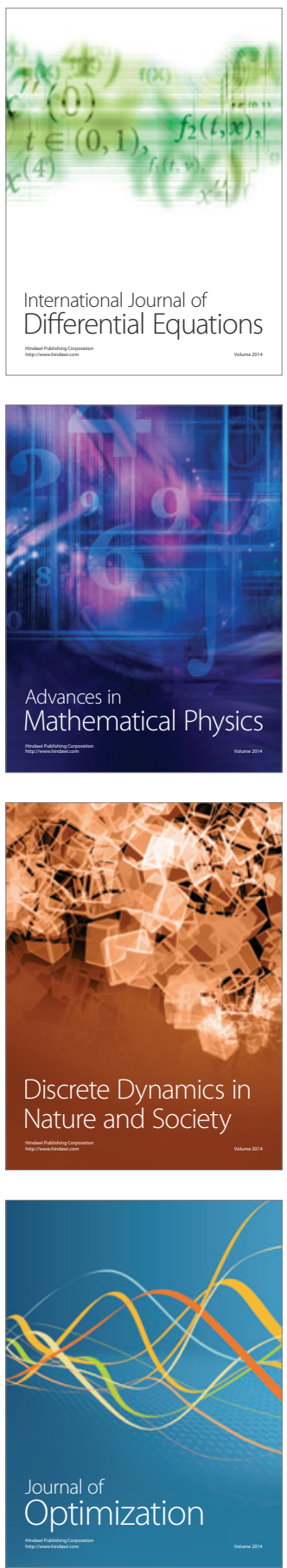